\title{
Heart disease, clinical proteomics and mass spectrometry
}

\author{
Brian A. Stanley ${ }^{\mathrm{b}, \mathrm{c}, * *}$, Rebekah L. Gundry ${ }^{\mathrm{a}, * *}$, Robert J. Cotter ${ }^{\mathrm{a}}$ and Jennifer E. Van Eyk ${ }^{\mathrm{b}, \mathrm{c}, *}$ \\ ${ }^{a}$ Department of Pharmacology, Johns Hopkins University, Baltimore, MD, USA \\ ${ }^{\mathrm{b}}$ Department of Medicine, Johns Hopkins University, Baltimore, MD, USA \\ ${ }^{\mathrm{c}}$ Department of Physiology, Queen's University, Kingston, Ontario, Canada
}

\begin{abstract}
Heart disease is the leading cause of mortality and morbidity in the world. As such, biomarkers are needed for the diagnosis, prognosis, therapeutic monitoring and risk stratification of acute injury (acute myocardial infarction (AMI)) and chronic disease (heart failure). The procedure for biomarker development involves the discovery, validation, and translation into clinical practice of a panel of candidate proteins to monitor risk of heart disease. Two types of biomarkers are possible; heart-specific and cardiovascular pulmonary system monitoring markers. Here we review the use of MS in the process of cardiac biomarker discovery and validation by proteomic analysis of cardiac myocytes/ tissue or serum/plasma. An example of the use of MS in biomarker discovery is given in which the albumin binding protein sub-proteome was examined using MALDI-TOF MS/MS. Additionally, an example of MS in protein validation is given using affinity surface enhanced laser desorption ionization (SELDI) to monitor the disease-induced post-translational modification and the ternary status of myoctye-originating protein, cardiac troponin I in serum.
\end{abstract}

Abbreviations: AMI, acute myocardial infarctions; BNP, B-type natriuretic peptide; HF, heart failure; MS, mass spectrometry; IEC, ion exchange chromatography; SEC, size-exclusion chromatography; 2D-LC, two-dimensional liquid chromatography; RP-HPLC, reversed-phase high performance liquid chromatography; SELDI, surface enhanced laser desorption ionization; TnI, troponin I; TnT, troponin T; MS/MS, tandem mass spectrometry; TOF/TOF, tandem time-of-flight; PTM, post-translational modification; PMF, peptide mass fingerprint; MALDI-TOF MS, matrix assisted laser desorption ionization - time-of-flight mass spectrometer.

\section{Introduction}

Heart disease is the leading cause of mortality in the world [5] and as such it contributes to high medical costs, lost work productivity and great personal loss. Functionally, heart disease is the inability of the heart to pump sufficient blood to meet the metabolic needs of the body. It can occur quickly, as with an acute myocardial infarction (AMI), or progress slowly over years as with chronic heart failure (HF) (Fig. 1). In each

*Corresponding author: J. E. Van Eyk, 602 Mason F. Lord Bldg., Bayview Campus, Johns Hopkins University, Baltimore MD, 21224, USA. Tel: +1 410550 8510; Fax: +1 410550 8512; E-mail: jvaneyk1@jhmi.edu.

${ }^{* *}$ Denotes equal contribution to paper. case, the heart is unable to efficiently or effectively beat (contract) making it more difficult to maintain cardiac output.

The ability of the heart to beat resides within the cardiac myocytes, the major cell type of the heart. Changes to the myoctye proteome (the protein complement of these cardiac contractile cells) collectively produce the pathological phenotype of the diseased heart. Possible protein changes include any combination of novel or altered expression, induction or elimination of post-translational modifications (i.e. phosphorylation), or isoform expression differences. Individually a change may be benign, compensatory or detrimental to the overall function of the myoctye. Thus, the detection, identification and characterization of changes to the proteome occurring during the onset and develop- 


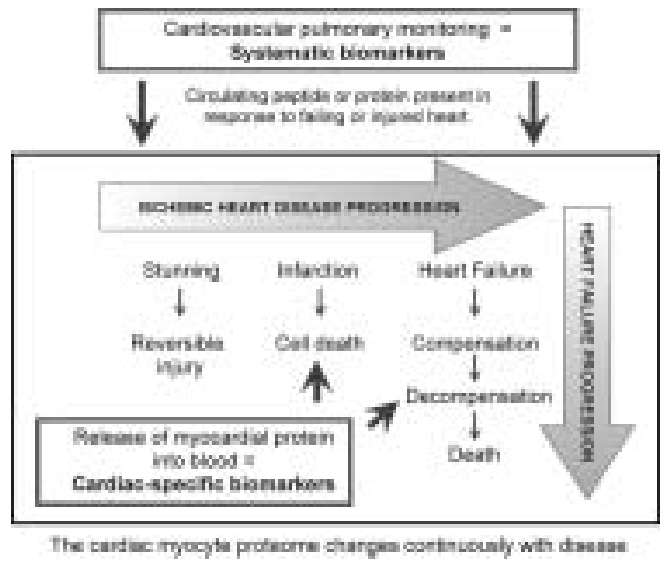

Fig. 1. Schematic representation of the progression of acute ischemic heart disease and chronic heart failure. With mild ischemia/reperfusion injury the myoctyes are reversibly injured (stunning) and with time the heart cells repair themselves and full function is restored. Increasing severity of ischemia will eventually result in myoctye cell death and myocardial infarction. Of the patients that survive a myocardial infraction, approximately $30 \%$ will go on to develop heart failure. In the early stages of heart failure, the cardiac myoctye is injured but is able to compensate so that cardiac output is maintained. At some point, perhaps due to a second injury, the heart decompensates, cell death occurs and cardiac output drops. Upon cell death, the myocardial proteins are released into the blood. Detection of these myoctye-originating proteins, and any of their disease-induced changes (new PTM or novel expressed proteins) are potential cardiac specific biomarkers. On the other hand, changes in the circulating proteome, peptides and proteins that are normally in blood or are present as signaling moieties are also potential biomarkers. In this case, detection and monitoring of the serum proteins and peptides may reflect the response of the cardiovascular pulmonary systems.

ment of heart disease will provide both (i) insight into the underlying molecular mechanism and (ii) potential biomarkers that are specific for the monitoring and assessment of the status of the heart (i.e. heart specific). Upon myocyte death, cellular proteins are released into the blood where their detection is possible. Furthermore, direct analysis of the blood (and potentially other body fluids) can also provide information about the heart and its interaction with the other organ systems within the body (cardiovascular pulmonary monitoring). Direct observation, quantification and characterization of circulating peptides and proteins can hopefully provide insight into the holistic response of the injured or failing heart. Although yet to be exploited, these biomarkers should be able to monitor the interplay between the cardiovascular pulmonary systems which will be particularly important in these complex diseases.

Proteomics is a scientific approach that attempts to completely characterize the proteome (or subproteome) of a cell or tissue. Many different technologies can be used during the three major steps involved in proteomic analysis: sample preparation, protein (or peptide) separation, protein identification and characterization, and the technologies can be mixed and matched to meet the needs to answer any particular biological or clinical question. Although it is not possible with today's technology to completely characterize the proteome, new methods and strategies are continually being developed towards this goal. Mass spectrometry (MS) is a central tool used for monitoring, identifying and characterizing proteins. With an increasing ability to correctly characterize miniscule quantities and more complex mixtures of proteins and peptides, MS is quickly becoming a key tool in the discovery of alterations to the proteome. Additionally, because of its low detection limit and its ability for high throughput analyses, the possibility of using MS directly in clinical assays is also becoming a reality.

\section{Clinical proteomics}

Clinical proteomics is defined as the application of proteomics to the field of medicine. It encompasses the translation of proteomic detection technologies and strategies towards the production of diagnostics and therapeutics for the direct improvement of human health [17]. Specifically, changes observed in the proteome of an animal model or clinical subject can be utilized directly as biomarkers or used as the basis for the development of a pharmacological intervention. In the past, biomarker detection and quantification using a single candidate protein has been used exclusively to provide either a yes/no answer. However, the evolving strategy of choice is to use multiple candidate proteins, which together can provide more complete information. Within the context of both acute and chronic heart disease, because of the spectrum of the disease process, effective biomarkers are critically needed for diagnosis, risk stratification, prognosis and determining the effectiveness of therapeutic treatment. Eventually, given sufficient biomarkers, clinical proteomics will allow for the generation of a grid of information obtained from the patient, which will allow for a finer manipulation and individualized treatment for patients.

\section{Strategies for biomarker discovery}

Irrespective of whether one is using tissue, serum or other body fluids, there are several common steps 
involved in the development of a diagnostic marker. These are (i.) Discovery, (ii.) Validation and (iii.) Translation of the discovery into clinical practice (Fig. 2).

Within each of these steps, MS can play a vital role.

(i.) Discovery - Goal dependent options:

Depending on whether the goal is the discovery of a heart specific biomarker or a system monitoring biomarker dictates the milieu to be examined (i.e. tissue or serum/plasma), the species to analyze (human or animal models), as well as the set of proteomic tools to be utilized. For instance, to obtain a cardiac specific biomarker, the analysis of cardiac tissue is necessary due to the extremely low concentration of cardiac-originating proteins in serum/plasma. Because of the extensive dilution that occurs upon cardiac necrosis, cardiac-originating proteins typically exist at serum concentrations $<1 \mu \mathrm{g} / \mathrm{L}$, making it difficult, if not impossible to detect these proteins in serum using conventional "unbiased" proteomic technologies. However, the analysis of human cardiac tissue is also hampered by issues of accessibility and limited quantity (except in case of transplanted or rejected hearts) as well as patient to patient heterogeneity that exist due to a myriad of reasons including differences in drug treatment, stage of disease and the presence of other overlapping disease etiologies (e.g. diabetes). Because of the issues which plague human samples, animal models are often used as the primary means of cardiac-specific biomarker discovery. The ability to manipulate the type and extent of injury as well as measure invasive physiological measurement make animal models often the best choice. Although cardiac-originating proteins exist at extremely low concentrations in serum, a specific protein change associated with a disease (determined from tissue analysis) may be examined for in serum using enrichment techniques such as immunocapture.

The "unbiased" analysis of serum or plasma is the other route for the discovery of biomarkers [39]. However, in this case these proteins will most likely be biomarkers for cardiovascular pulmonary monitoring diagnostics due to the wide range of proteins present in serum/plasma. The advantage of utilizing serum/plasma as the milieu of interest is that it is more easily obtained than tissue, but unfortunately it is also more difficult to analyze due to the presence of highly abundant proteins such as albumin $(45-50 \mathrm{mg} / \mathrm{mL})$, which interfere with the detection of lower abundant proteins. This issue can be overcome through the efficient depletion of these high abundant proteins, including albumin $[1,7,16,29,35]$ from both serum and plasma using either salt precipitation or affinity based methods. However, these depletion methods are hampered by the nonspecific removal of other serum proteins [13] bound to albumin, which may be powerful biomarkers. As such, a new area of interest for biomarker discovery is the analysis of the proteins bound to albumin, which one might term "the albuminome" (See Example 1). The discovery of biomarkers in serum/plasma is often accomplished using animal models due to similar issues as with tissue, even though secondary analysis is also required to validate these markers in human disease. However, if one chooses to use patient serum, the patient to patient heterogeneity can be overcome through (a) increasing the number of patients to be studied, (b) analysis of at least two samples obtained at different times (stages of disease or intervention) so the same patient acts as their own internal control, or (c) the use of clinically well-characterized patients. Whenever possible it is reasonable to validate the presence of the potential tissue markers in the corresponding human disease tissue.

(i) Discovery - Technologies:

The analysis of a complex mixture such as the cardiac tissue proteome requires a combination of techniques and technologies that must first separate the protein or peptide mixture, and secondly determine the identity of the constituents. The most widely used protein separation technique for proteomics is two-dimensional gel electrophoresis (2-DE) because of its ability to display a large number of proteins simultaneously and differentially. 2-DE separates proteins based initially on pI (isoelectric focusing (IEF)) followed by molecular weight (via SDS PAGE). For less complex proteomes (i.e. those from simpler organisms or subsets of the proteins from a complex organism), the simpler one-dimensional gel electrophoresis (SDS PAGE, 1-DE) can be used, which separates proteins based on mass. In addition to gel-based methods a wide range of chromatographic methods are finding widespread use [23,26,27], including ion-exchange chromatography (IEC), size-exclusion chromatography (SEC), two-dimensional liquid chromatography (2D-LC) and reversed-phase high performance liquid chromatography (RP-HPLC) as well as a number of affinity based chromatography methods. Chromatography methods can be used for either proteins or peptides (achieved after digest of the protein mixture). The prime benefit of these methods is their applicability to both protein and peptide separations as well as their amenability to automation. Additionally, another high-throughput technique used for separation as well as protein pro- 


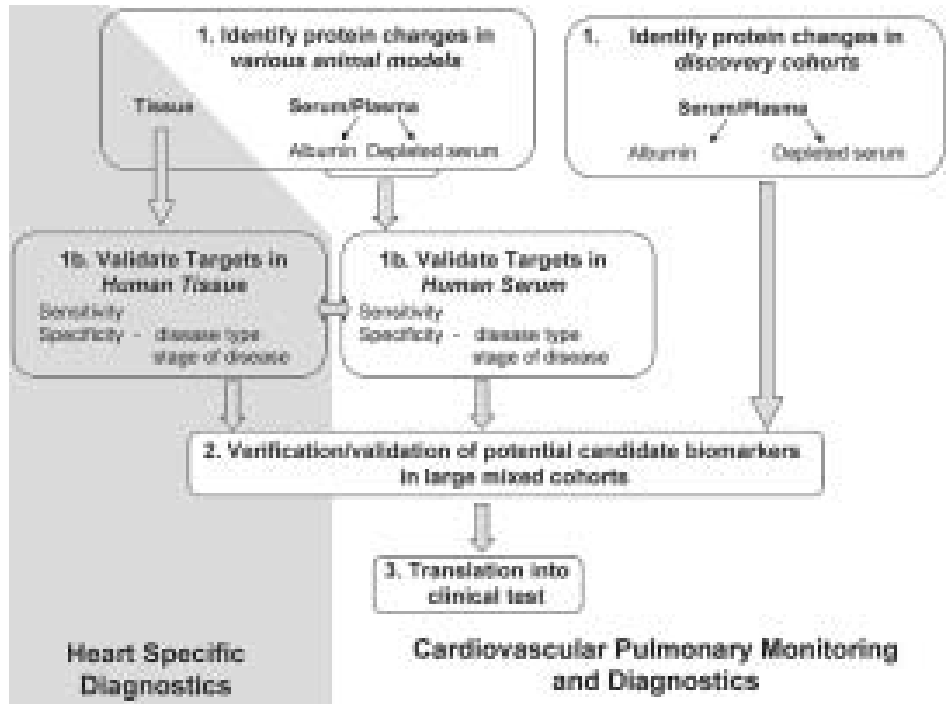

Fig. 2. Strategies for development of heart-specific diagnostics and cardiovascular pulmonary system monitoring and diagnostics. Analysis involves three steps (i.) Discovery, (ii.) Validation and (iii.) Translation of the discovery into clinical practice and the production of the diagnostic marker or panel.

filing is surface enhanced laser/desorption ionization (SELDI). SELDI offers an on-chip separation and capture of proteins based on a variety of chemical and biological matrices, coupling direct mass spectral analysis to chemical and affinity-based separations.

Methods for the separation and purification of proteins are best complemented by some means of protein identification - a crucial element of the discovery step. Mass spectrometry has become an invaluable technique of choice for both protein profiling as well as for positive protein identification. With the expansion of protein sequence databases and search engines designed for direct input of mass spectrometry data, the mass spectrometer can provide rapid and accurate identification of proteins for any number of species for which whole or partial genomes have been determined. Unlike earlier mass spectrometry methods used to identify chemical species from mass spectral libraries, the masses in a spectrum can now be compared directly with masses calculated for the peptide products of enzymatic digestion or the fragments expected from the sequence. For purified proteins and simple mixtures, it is common to use peptide mass fingerprinting (PMF) to identify the proteins [18]. In this approach, the masses resulting from the whole or partially purified peptide mixture following tryptic digestion are used to interrogate the database, usually restricted to proteins with a range about the measured intact protein mass. In addition to their identity, changes in the expected mass resulting from post-translational modifications (such as phosphorylation, sulfation, acetylation, etc.) and other post-processing such as cleavage of methionine residues are often observable.

For more complex mixtures, like those more commonly found in proteomics studies, positive identification cannot be provided by PMF alone and sequence tags are required. A sequence tag is generally some continuous stretch of amino acid sequence within one or more of the peptide fragments used for mass fingerprinting. The sequence tag provides considerably higher specificity than PMF alone when used in conjunction with the intact fragment mass. Such sequence tags can be acquired by any one of several types of tandem mass spectrometers that include quadrupole ion traps, ion trap/time-of-flight hybrids and tandem timeof-flight (or TOF/TOF). In tandem mass spectrometry (MS/MS), a single peptide mass located in the MS spectrum is isolated in the mass spectrometer and the peptide is fragmented. Fragmentation may occur by metastable decay (from the ion's own internal energy) or be induced by collisions with a gas. When carried out on a tandem time-of-flight, MS/MS (with or without a collision gas) analysis of fragments has been made considerably easier by the development of the curved field reflectron (CFR) [10]. Unlike the single and dual stage reflectrons used in most commercial time-of-flight mass spectrometers, the CFR focuses all of the "product" (sequence) ions simultaneously so that an MS/MS spectrum can be obtained in the same time it takes to acquire the first dimension MS spectrum. 
In current studies, protein identification is achieved by a reiterated identification approach that combines the information garnered from sequence tags and reverse-PMF. In this strategy, MS/MS is performed on every peptide with sufficient signal to noise. The sequence information from each peptide is then searched against an on-line database using engines such as Mascot, XProteo or Protein Prospector. The redundant nature of the data provided by multiple, orthogonal data matching to a particular protein ultimately confirms a protein's identity(s). Peptides for which insufficient sequence information is available by MS/MS are checked by reverse-PMF. In this method, the predicted tryptic peptides of the proteins identified by MS/MS are compared to the masses of the tryptic peptides observed in the sample. In this way, peptides not matched to the identified proteins can indicate the presence of additional proteins.

As the field of proteomics progresses and protein identifications become a required complement to protein profiles, techniques such as mass spectrometry will prove to be invaluable. Moreover, although single dimension and quantitative MS may be the best approach to high throughput screening in differential protein expression studies, MS/MS will likely need to be routinely applied in the identification and validation stages.

\section{Example 1 - MS used in biomarker discovery}

\subsection{Overview of chronic heart disease}

Following an acute myocardial infarction (AMI), approximately $30 \%$ of patients will develop HF within 2 years. The primary cause of HF is due to detrimental changes that occur to the myocardium following an AMI; however, HF is a chronic, multi-factorial disease in which different triggers including genetics and environmental factors interplay resulting in a variable rate and severity of disease progression. Initially, the heart and myocytes are able to compensate for the cellular death and maintain cardiac output. However, with time and further stress, the heart is unable to meet the physiological demands of the body and symptoms such as shortness of breath and lethargy result. Eventually, the heart deteriorates to the point that cell death occurs and myocytes are lost. There currently is no cure for heart failure but rather only the treatment of the physiological side effects that occur due to inefficient heart function. The prognosis for a patient diagnosed with HF is poorthey have a 6 to 9 times higher rate of sudden cardiac death compared to the general population and greater than $85 \%$ of patients will die within $8-12$ years [5]. As such, there exists a need to discover biomarkers for HF, which will allow early diagnosis and the ability to monitor the progression and development of this disease.

A biomarker currently used for heart failure is B-type natriuretic peptide (BNP), a hormone released from the heart in response to increased haemodynamic pressure. BNP acts to reduce total blood volume by affecting a large number of organs within the cardiovascular system such that cardiovascular wall stress is reduced. The tight interplay between the cardiovascular and pulmonary system occurs mainly through blood borne hormones, proteins and peptides. It is the detection of these "information" triggers, which will allow us to have insight into the status of the entire cardiovascular system. Although these triggers do not provide direct information about the heart, they should provide key mechanistic information with regards to the cardiovascular-pulmonary systems. What is not clear is whether detection of these "cardiovascular system" information biomarkers will be sufficiently specific for a disease state to be of use in the clinical arena. However specific or non-specific these biomarkers might be, they will still provide important information about the overall status of the patient.

\subsection{Search for CV-Biomarkers in the albuminome}

As mentioned in the discovery section, in order to study the low abundant proteins in serum, it is necessary to first remove highly abundant proteins such as albumin prior to a proteomics study. Multiple methods to remove albumin have been employed in different studies using either blue textiles dyes (e.g. Cibacron blue), affinity chromatography, or centrifugal filtration [1, 29,35]. However, albumin functions as a "molecular sponge" binding proteins, lipids, and small molecules in the intracellular space. As such, other proteins are removed along with albumin during its removal. We predict that those proteins bound to albumin (the albuminome) will be altered in patients with heart failure. That is, in the disease state, either newly released proteins/peptides will bind to albumin or those proteins/peptides that normally bind albumin will bind at a higher concentration due to their increased concentration in serum. Following the methodology mentioned in the discovery section, our first goal was to identify all proteins in the albuminome using a swine model of heart failure. 


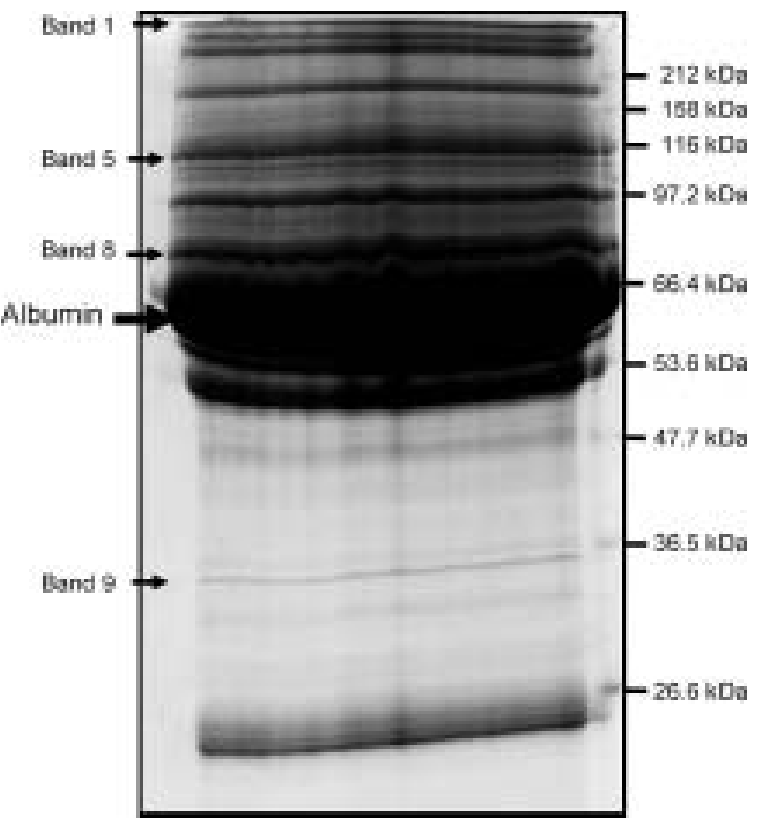

Fig. 3. SDS-PAGE analysis of albuminome and potential albumin-binding proteins. Albumin and any associated proteins were extracted from control swine serum using affinity chromatography, Cibacron blue. The commassie blue stained $12 \%$ gel is shown, the arrow indicates the band containing primarily albumin. The proteins were purified from $100 \mu \mathrm{L}$ of serum.

In efforts to characterize the albuminome, swine serum $(40 \mu \mathrm{L})$ was mixed with $\mathrm{NH}_{4} \mathrm{HCO}_{3}(40 \mu \mathrm{L})$ and incubated with $100 \mu \mathrm{L}$ Cibacron blue beads (Pharmacia, NY). The beads were removed and boiled in sodium dodecyl sulfate (SDS) to remove any bound proteins from the beads. The resulting supernatant was then run on $12 \%$ SDS-PAGE. Proteins bands were cut out and incubated in a trypsin digest solution $(10 \mathrm{mg} / \mathrm{mL}$ trypsin in $25 \mathrm{mM} \mathrm{NH}_{4} \mathrm{HCO}_{3}$ ). The resultant tryptic peptides were extracted with an acetonitrile-trifluoroacetic acid (TFA) solution and subsequently lyophilized. In preparation for MALDI-TOF MS, the samples were resuspended in $2 \mu \mathrm{L} 0.1 \%$ TFA and spotted via a sandwich method onto the MALDI-TOF probe with a saturated solution of $\alpha$-cyano-4-hydroxycinnamic acid (CHCA) in $50 \%$ ethanol/water. All spectra of the tryptic digest were acquired using a Kratos (Manchester, UK) Axima CFR high performance MALDI-TOF. For each gel band, a mass spectrum of the tryptic fragments was obtained (Fig. 3).

Subsequently, MS/MS (without collision gas) was performed on all peaks with reasonable signal-to-noise ratio (Fig. 4). Fragment masses from the MS/MS spectra of each peptide were typed into the Xproteo online search engine (http://xproteo.com) in order to search protein databases for protein identity. When possible, peptides with insufficient fragment information from the MS/MS spectra were assigned to proteins via reverse-PMF. In this method, the predicted tryptic peptides of the proteins identified by tandem MS were compared to the masses of the tryptic peptides in the sample. Table 1 shows the assignment of the proteins identified from their respective bands. As illustrated in Table 1, multiple sequence tags were obtained for most proteins.

Inter-alpha trypsin inhibitor heavy chains 1 and 2 are observed in band 1 . While the molecular weight of band 1 appears to be significantly higher than the $212 \mathrm{kDa}$ marker, the sum of both chains together is approximately $205 \mathrm{kDa}$. This molecular weight discrepancy could be due to the fact that these two chains are connected by a protein-gylcosaminoglycan-protein (PGP) crosslinker [31]. While the molecular weight of the crosslinker is not enough to account for the difference between the observed and calculated molecular weights of the complex, it is known that this crosslinker is resistant to denaturation. Therefore, it is predicted that these two chains are intact within the gel and their confirmation within the bound complex contributes to the observed molecular weight. The results from these efforts illustrate that the methods described here can be used to routinely and effectively identify proteins in mixtures. As with all proteomics studies, following protein identification, any potential biomarkers must be validated in the final stage of biomarker development.

ii) Validation

The goal of the validation stage (Fig. 2) is to determine which single (or group of) biomarker(s) achieves the highest sensitivity and specificity for a particular clinical diagnosis of a disease state. The biomarker(s) may be selected from either existing candidate proteins or from those identified as potential biomarkers (or profile) during the discovery stage. In either case, the potential biomarker is compared against the known biomarkers in one or more validation studies comprised of large, well-phenotyped cohorts. This allows one to rule out the many false positive biomarkers which may be identified during the discovery stage as a result of a small sample size.

Validation is a critical step as it serves to weed in (or out) both the false positive as well as other less relevant (less sensitive or specific) markers based on their sensitivity and specificity as clinical tools, thus allowing for a determination of the most effective and efficient biomarker(s). Biomarker validation is not a trivial exercise and is dependent on both the choice of 
Table 1

Proteins identified in the albuminome. Corresponding band from $12 \%$ gel (Fig. 3), molecular weight, and fragments observed are listed. Fragment masses for which sufficient fragment information was obtained are in bold. Other masses were assigned to protein via reverse peptide mass fingerprinting

\begin{tabular}{|c|c|c|c|}
\hline Protein & Band & MW (kDa) & Fragments observed \\
\hline $\begin{array}{l}\text { Inter-alpha typsin } \\
\text { inhibitor (H1) Precursor }\end{array}$ & 1 & 100.4 & $\begin{array}{l}\text { 787.0, 829.0, 866.9, 931.2, 939.8, 1173.7, 1205.6, 1247.6, 1469.9, 1473.1, } \\
1629.8,2037.6\end{array}$ \\
\hline $\begin{array}{l}\text { Inter-alpha typsin } \\
\text { inhibitor }(\mathrm{H} 2) \text { Precursor }\end{array}$ & 1 & 104.6 & $\begin{array}{l}\text { 821.0, } 891.9,907.9,975.8, \mathbf{1 3 3 7 . 6}, \mathbf{1 4 5 2 . 4}, 1582.3, \mathbf{1 7 4 5 . 1}, 1803.9,1807.9 \text {, } \\
2239.4\end{array}$ \\
\hline $\begin{array}{l}\text { Complement Component } \\
\text { C3 }\end{array}$ & 5 & 186.81 & $\begin{array}{l}\text { 787.2, 833.3, 845.3, 860.3, 868.4, 887.3, } 900.4, \mathbf{9 1 2 . 4}, 959.4,1053.5, \mathbf{1 0 8 3 . 5} \text {, } \\
1099.6, \mathbf{1 1 2 6 . 5}, \mathbf{1 1 4 3 . 5}, 1211.6, \mathbf{1 2 9 0 . 6}, 1457.8,1669.8,1683.9,2170.4\end{array}$ \\
\hline $\begin{array}{l}\text { Fibrinogen Alpha chain } \\
\text { - Fibrinopeptide A }\end{array}$ & 8 & 1.76 & $1091.4,1761.6$ \\
\hline $\begin{array}{l}\text { Fibrinogen - A-alpha } \\
\text { chain }\end{array}$ & 8 & 47.33 & $\begin{array}{l}\text { 702.3, 848.1, 1100.5, 1107.4, 1145.5, 1183.5, 1296.5, 1316.5, 1379.5, } \\
1579.7\end{array}$ \\
\hline Thrombopoietin & 8 & 2.9 & 870.4 \\
\hline $\begin{array}{l}\text { Apolipoprotein-E } \\
\text { precursor }\end{array}$ & 9 & 36.58 & $\begin{array}{l}\text { 886.4, 899.3, 958.4, 968.4, 990.5, 1033.4, } 1077.4, \mathbf{1 1 4 6 . 5}, 1274.5, \mathbf{1 5 4 2 . 6} \text {, } \\
1743.6, \mathbf{2 2 8 8 . 8}\end{array}$ \\
\hline
\end{tabular}

the clinical cohort as well as the robustness of the clinical phenotyping. For complex multi-factorial diseases such as heart disease, validation may require that the biomarker be tested against a series of different cohorts. For instance, the first validation cohort can be confined to patients with "clear cut" diagnosis of the disease vs. those who may have the disease. That is, if you are interested in identifying a new biomarker for the early detection of myocardial infarction, one potential cohort would be all patients entering into an emergency ward who have chest pain vs. those who definitively have had an AMI. The validity of the potential biomarker would then be determined by comparing its ability to both detect those patients diagnosed with an AMI (and possibly angina) using conventional means vs. detecting an AMI in previously "AMI negative" patients. Of course, with heart disease, absolute diagnosis is difficult, but detailed phenotyping and the use of surrogate markers may help in the diagnosis. Heart disease is also complicated due to the close interplay between numerous organ systems, the influence of therapeutics which many heart patients are taking, and overlapping diseases such as diabetes or COPD which make it difficult to determine the absolute specificity of a biomarker. Therefore, it may become important to assay potential biomarkers against a second (or even third) validation cohort in order to eliminate any effect from confounding factors that could lead to either a false negative or positive result. In using multiple cohorts, the goal is to eliminate or at least identify biomarkers that can be labeled as "general illness markers" that overlap different diseases. A key point to remember, however, is that the ability to correctly identify patients is based both on the quality and accuracy of the clinic diagnosis as well as the sensitivity of the technology used to detect the potential biomarker in serum.

ii) Validation: Technology

There are currently only a limited number of commercially available technologies that are appropriate for screening large cohorts in a quick effective manner and several of these are based on MS. In each of these assays, the essential mechanism provides for both the capture and detection of the potential biomarker. Generally, the analyte is captured using a specific antibody, but certainly other binding moieties (peptide meditics, aptamers, protein binding partners) are also available. The detection and/or quantification of the biomarker can be accomplished using either a second antibody (or chemical moiety) to the same protein (currently the most common setup for clinical laboratory immunoassays) or other physical means including mass spectrometry. The development and testing of protein chips for biomarker analysis is a rapidly growing field, and how they eventually will be incorporated into the diagnostics arena is still unknown $[8,20,25]$. The development of many of the components of protein arrays such as immobilization strategies [24]; fabrication and support [30,32]; sensors [28,37] and the use of microfluidic systems [6] or capillary electrophoresis/MS methods [33] have to be enhanced before protein chips can become a mainstream technology. In cardiac disease, the identification and quantification of apoA-1 and II and their PTM forms [11] is the first study to demonstrate the feasibility of using protein chips for biomarker validation. Ultimately, irrespective of the tool used, for effective biomarker validation, the instru- 


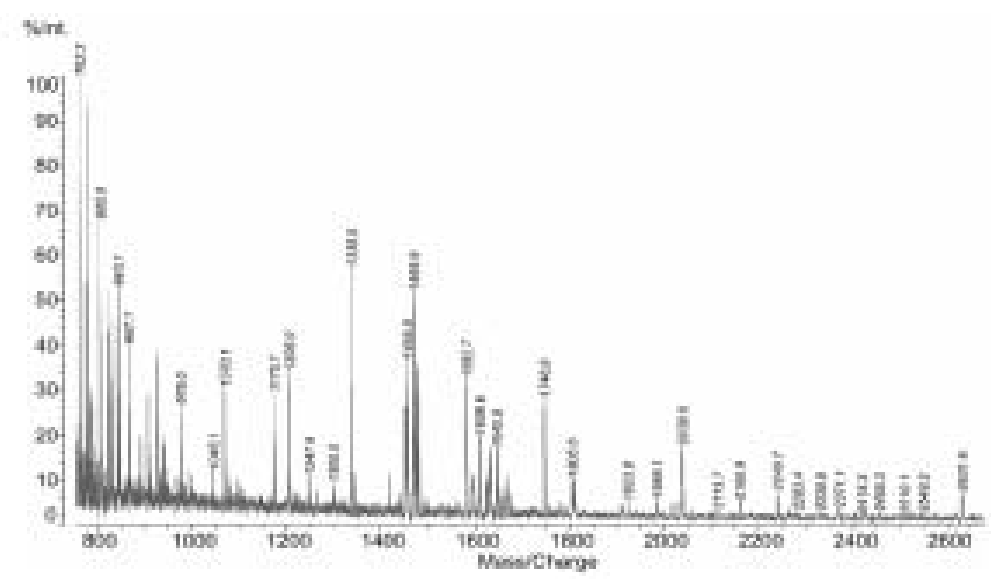

B) MS/MS spectrum and amine acid sequence obtained from Kratos Aximn CFR MALDI-TOF instrument

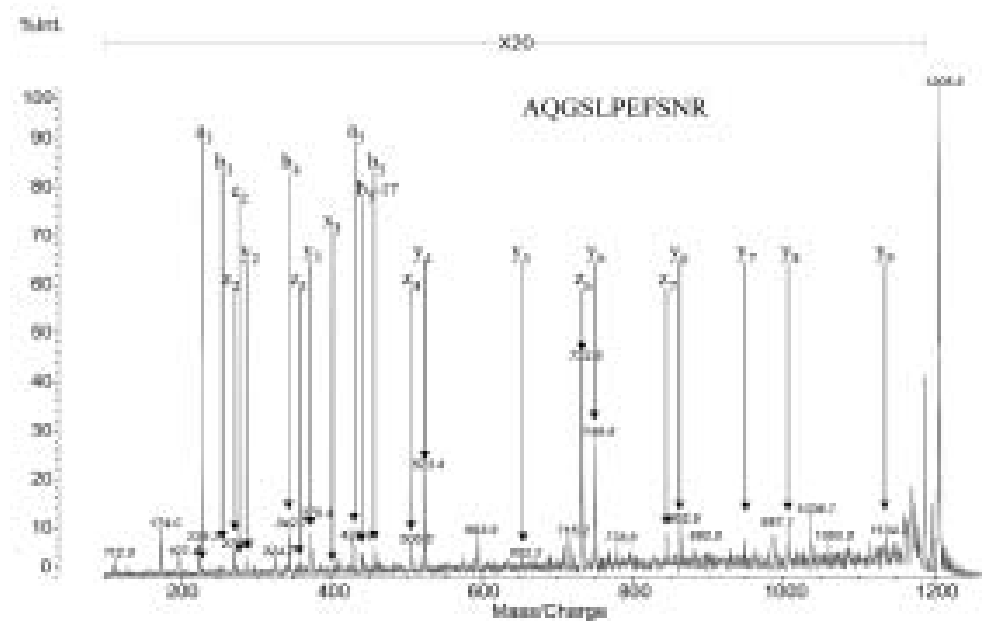

Fig. 4. Mass spectrometry data from the albuminome. Panel A shows the MALDI-TOF MS spectrum of tryptic digest of largest band (band 1) from $12 \%$ gel shown in Fig. 3. Panel B shows the Tandem MS spectrum of peak 1205.8 Da from the tryptic digest of band 1 of the $12 \%$ SDS PAGE gel shown in Fig. 3. The fragment ions are labeled appropriately. Both spectra were obtained on the Kratos Axima CFR MALDI-TOF instrument. The spectrum of the tryptic peptide masses was obtained first and then sequence data was obtained by MS/MS. Protein identifications were determined based on the MS/MS data and then reverse PMF was used to ensure that there were no additional contaminating proteins.

mentation needs to be fast and have the ability to effectively screen for a single or possibly multiple biomarkers with high analytical sensitivity and precision. Additionally, this must be accomplished using minuscule quantities of sample as the quantity available from the validation cohorts is limited. MS can be used as a sensor either to monitor whole mass of the proteins such as with SELDI or to identify immobilized protein(s) by PMF and MS/MS. Both approaches have their value and limitations which are illustrated in Example 2.

iii) Translation.
Translation involves the transfer of a diagnostic test from theory into everyday clinical practice. The exact format for a clinical test in which multiple markers are used to improve patient diagnosis is not clear. Certainly the use of immunoassays, protein chip arrays with detection based on MS, or other methods are possible and should be robust enough for the clinical every day setting. However, issues regarding standardization, reproducibility, robustness and cost will play a role in determining the final form for many clinical assays. What is clear, however, is that there needs to be communication between the scientists and clinicians developing such 

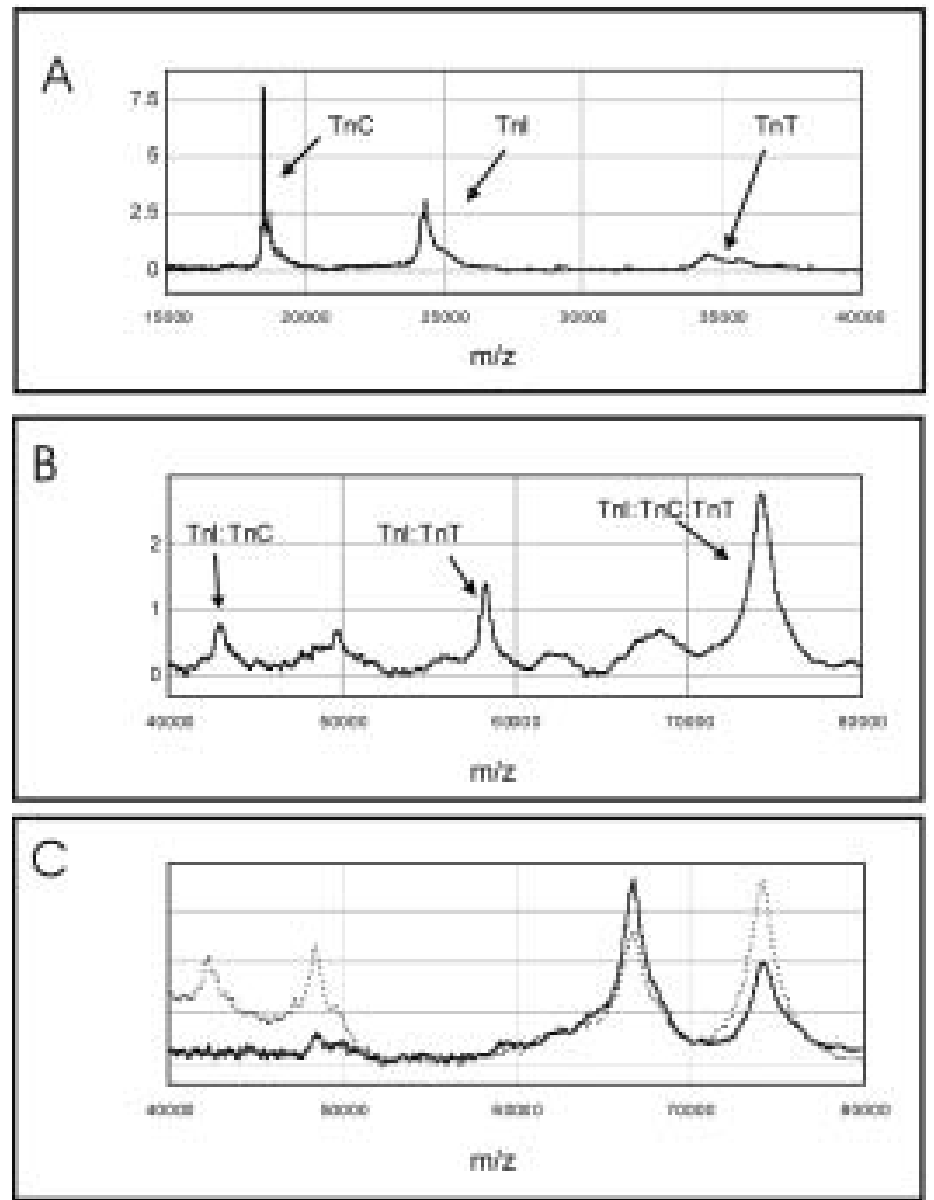

Fig. 5. Mass spectrometry data from TnI capture using SELDI-TOF MS. Panel A shows the low mass spectrum from mouse tissue extract, the peaks corresponding to TnC, TnI, and TnT are labeled. Panel B shows high mass spectrum from mouse tissue, the peaks corresponding to the TnI:TnC and TnI:TnT dimers as well as ternary troponin are shown. Panel C shows high mass spectra from human tissue (dashed line) and human serum following an AMI (solid line).

biomarker panels, the clinical chemists who will use these tests on a routine basis, and the physicians who will use the data generated by the tests to diagnose their patients. Interaction with commercial companies to encourage technological developments will be necessary. Finally, education of cardiologists and emergency physicians in the limitations as well as the advantages of the multi-marker approaches will be essential.

\section{Example 2 - MS used in biomarker validation}

\subsection{Overview of Acute Myocardial Ischemia (acute heart disease)}

Blockage of a coronary artery will lead to a partial or complete reduction in blood flow (ischemia) to a specific region of the heart. If adequate reperfusion to the ischemic region does not occur rapidly, cardiac myocytes will undergo necrosis and die. The severity of an AMI (and risk to the patient) depends on a multitude of factors including the size of the region that the artery supplies (size of the ischemic region/zone), the duration of the ischemic event, as well as the effectiveness of reperfusion. Hence, there is a need for a quick, accurate and sensitive biomarker assay for diagnosis of AMI in order to start reperfusion therapy as early as possible allowing improved salvage of the myocardium.

Upon cellular death, myocytes within the ischemic zone release their protein complement into the blood. The detection of myoctye-originating proteins forms the basis for many of the biomarkers in use for the diagnosis of AMI. The current gold standard for diagnosing AMI utilizes the cardiac muscle specific iso- 
forms of two key contractile proteins, troponin I (TnI) and troponin $\mathrm{T}(\mathrm{TnT})$ [2,3]. TnI and TnT along with troponin $\mathrm{C}(\mathrm{TnC})$ form the ternary complex troponin which functions as the primary regulator of cardiac muscle contraction. Because cardiac-specific TnI and TnT are normally only expressed within cardiac myocytes, in theory, they should only be present in blood following necrosis of cardiac myocytes. Over the past decade, these biomarkers have lead to improvements in the ability to diagnosis an AMI, which has resulted in quicker treatment and consequently a greater likelihood of survival. However, recent evidence by our lab [9] has demonstrated the need for assays with increased sensitivity for TnI which would ultimately allow for an immediate determination of a patient's risk for an AMI.

In addition to its mere presence, the "form" of TnI or "its physical status" present in serum may be a critical factor in the diagnosis of cardiac disease. TnI can exist in multiple forms as a result of PTMs such as phosphorylation [4], oxidation [38], and site specific proteolysis $[19,21]$ and importantly, these states appear to be altered with disease. It is possible that the diseaseinduced PTM of TnI can alter its interaction with the other subunits of troponin ( $\mathrm{TnC}$ and $\mathrm{TnT}$ ) in tissue and presumably in serum. Already there is evidence suggesting that instead of existing as a monomer, TnI exists in serum as a TnI:TnC dimer [19,22] and/or possibly the ternary TnC:TnI:TnT complex $[14,15]$. Therefore, it is important to determine the PTM and ternary status of TnI in serum. However, due to its insolubility and basic pI (pI 9.5) TnI is difficult to analyze using conventional techniques [34]. Therefore, our laboratory has used an intriguing experimental method that uses MS to determine the status of TnI [22]. cTnI is immunocaptured by an antibody covalently bound to sepharose beads. The cTnI is subsequently digested with trypsin while it is still bound to the sepharose bead. The antibody was resistant to proteolysis by trypsin and as such, it did not interfere with the ability to obtain amino acid sequence coverage for TnI, TnT and $\mathrm{TnC}$ from tissue and coverage for $\mathrm{TnI}$ and $\mathrm{TnC}$ from serum. This was the first time that the IC complex was directly observed in serum. One limitation of the onbead method is that the MS may only detect a portion of the tryptic fragments from the proteins bound to TnI. Therefore, post-translational modifications of TnI or its binding partners may not be observed. However, the absence of a peptide fragment in the MS spectrum does not necessitate its absence or modification in the intact protein. As such, an additional immunocapture method that utilizes SELDI-TOF MS is necessary to directly determine the binding partners of $\mathrm{TnI}$ and potentially any disease-induced PTMs.

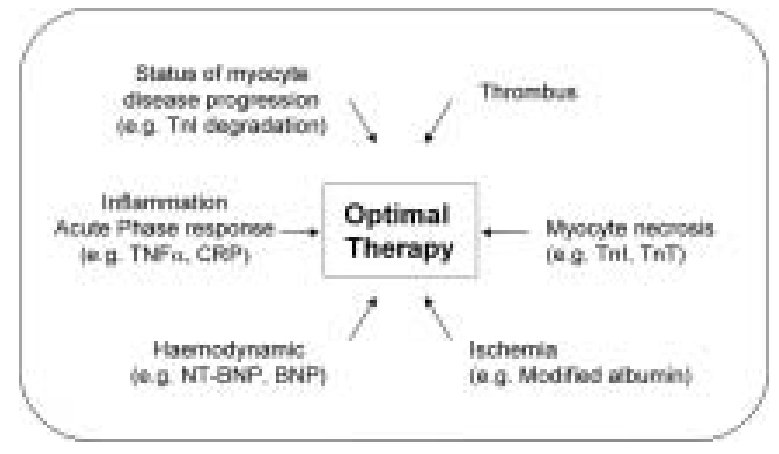

Fig. 6. Potential biomarker areas that will improve clinical treatment.

\subsection{Using SELDI-TOF MS to determine TnI binding partners}

A SELDI-MS protein chip is comprised of a MALDI target surface coated with a specific binding chemistry or biology, thereby allowing one to probe a particular subproteome or protein subset. Diverse surfaces ranging from a quaternary amine surface that functions as an anion exchanger to that of a nitriloacetic acid (NTA) surface which allows for immobilized metal affinity capture are available [36]. Of particular interest is the ability to immunocapture a protein to an antibody bound via a surface pre-activated with carbonyl diimidazole (RS100). Utilizing the RS100 chip, it was possible to capture TnI and those proteins bound to it directly to a SELDI target for MS analysis. These results illustrate the ideal progression from discovery to validation for any potential candidate biomarker. In order to validate the presence of $\mathrm{TnI}$ in the sera of a patient following an AMI, the initial observance of TnI in mouse tissue served as a critical step in the initial biomarker discovery stage.

The validation of the marker was then subsequently validated in human tissue and ultimately in sera obtained from a patient with an AMI (Fig. 5).

\subsection{Capture of troponin via troponin I}

The myocardium from an end-stage heart failure patient (who had previously experienced an AMI) was obtained at time of their heart transplantation. Frozen mouse myocardium was obtained from Pel-Freez laboratories (Pel-Freez, Brown Deer, WI). Serum was obtained from a patient with a diagnosed AMI following their presentation in the emergency department. Frozen mouse and human left ventricle were homogenized in extraction buffer $(20 \mathrm{mM}$ Hepes $\mathrm{pH} 7.4$, $0.25 \mathrm{mM}$ PMSF, $1 \mu \mathrm{M}$ leupeptin, $1 \mu \mathrm{M}$ pepstatin, 
$0.3 \mu \mathrm{M}$ aprotinin, $2.5 \mathrm{mM}$ EDTA, $0.2 \mathrm{mM} \mathrm{Na}_{3} \mathrm{VO}_{4}$, $50 \mathrm{mM} \mathrm{NaF}$ ). For mouse tissue, a single extraction using 5 volumes $(\mathrm{mL} / \mathrm{g})$ of extraction buffer was used whereas for human tissue, 2 consecutive extractions using 4 volumes were used. The solubilized myocardial proteins were centrifuged at $18000 \times \mathrm{g}$ for $15 \mathrm{~min}-$ utes with supernatant (myocardial extract) removed and stored at $-80^{\circ} \mathrm{C}$ until analyzed. The RS100 antibodychip (Ciphergen, Fremont, CA) was prepared by incubating $5 \mu \mathrm{g}$ of TnI antibody (mAb 8I-7, Spectral Diagnostics, Toronto, Canada) in $37.5 \mathrm{mM}$ NaHCO3 (pH 9.2 on the RS100 chip at $4{ }^{\circ} \mathrm{C}$ for 16 HRS. Nonspecific protein binding sites were blocked by incubating the target for $1 \mathrm{hr}$ at room temperature in $50 \mu \mathrm{M}$ BSA in PBS, pH 7.2. The chip was then washed with PBS. The myocardial extract was diluted 4 fold (50 ul was added to $150 \mu \mathrm{l}$ ) in the binding buffer which consisted of PBS; pH 7.2, 0.1\% TX-100 and incubated on the chip mounted to the Bioprocessor adaptor $(\mathrm{Ci}-$ phergen) for 2 hours at room temperature. A series of $30 \mathrm{sec}$. washes of PBS ( $\mathrm{pH} 7.2)+0.1 \%$ TX100; PBS (pH 7.2); $50 \mathrm{mM}$ Tris, 1 M Urea, 0.1\% CHAPS, $0.5 \mathrm{M} \mathrm{NaCl}$, pH 7.2 followed by a 2 minute wash in PBS and finally $\mathrm{H}_{2} \mathrm{O}$. The antibody-chip was then air dried for 5 minutes and $25 \mu \mathrm{g}$ of sinapinic acid matrix (50\% Acetonitrile, 50\% TFA) was added. Both low mass and high mass spectra were acquired using the ProteinChip ${ }^{\circledR}$ Reader (Proteinchip ${ }^{\circledR}$ System II; Ciphergen) and analyzed using the ProteinChip ${ }^{\circledR}$ Software (Ciphergen). Low and high mass range spectra for mouse tissue are shown as Figs 5(A) and 5(B) respectively, whereas the high mass spectra for human tissue and serum are shown as Fig. 5(C). Three peaks can be observed in Fig. 5(A) corresponding to the three individual members of troponin complex (TnC, TnI, and TnT) in mouse tissue. Additionally, in the mouse myocardium, masses were observed that matched the mass of TnI:TnT and TnI:TnC dimers and the trimer consisting of TnT:TnT:TnC (Fig. 5(B)). In human serum and tissue, there are two mass peaks observed around 77,000 Da which may represent the intact and degraded forms of the troponin complex although further characterization is required. In either case, these data illustrate the power of SELDI-TOF as both a powerful tool for the discovery of novel biomarker binding partners as well as a tool for the validation of a cardiac biomarker in serum.

As demonstrated in this example, a feature of SELDI-TOF MS is its ability to observe protein degradation products or other binding partners in serum directly. Many myocyte proteins including TnI undergo progressive and selective ischemic-induced modifications including proteolysis. Ding et al. have demonstrated that low molecular weight proteolytic fragments from TnI are directly observable using SELDI-TOF MS [12]. However, detection of these disease induced modifications has yet to be exploited in diagnostics. These changes can complicate the identification of the proteins by potentially producing altered masses. Specifically, ischemic induced proteolysis of cTnI is at least in part responsible for the difference in sensitivity of the many different commercial immunoassays.

\section{Panels vs. single biomarkers and looking forward to the future}

In the past, monitoring heart disease has focused on the detection and quantification of a single analyte (i.e. cTnI and BNP) providing only a yes/no answer. Today, however there is a move towards adding increased specificity by monitoring or assessing multiple biomarkers - commonly referred to as a panel of biomarkers. In theory, a larger panel profiled will allow for a more accurately and uniquely defined status of a patient. As an illustration, one can envision combining a biomarker (or multiple markers) for AMI that are able to specifically assess 1) whether the myocardium is ischemic, 2) under haemodyamic pressure, 3) pulmonary capacity 4) reperfusion efficiency (ie whether angioplasty or TPA treatment was effective), 5) severity of ischemic injury, 6) risk stratification and 7) the prognosis of a second AMI (Fig. 6). By combining sound biomarker discovery and validation approaches such as those described here with advancements in MS technologies, the field of clinical proteomics can begin to fulfill its role in the clinical setting by providing efficient and cost-effective clinical assays.

\section{Acknowledgements}

This work was supported-by the Hopkins NHLBI Proteomics Grant, Contract NO-HV-28180. Many thanks to Irina Neverova, Heather Brown and Kathy Baer for their discussion and technical assistance.

\section{References}

[1] N. Ahmed, G. Barker et al., An approach to remove albumin for the proteomic analysis of low abundance biomarkers in human serum, Proteomics 3 (2003), 1980-1987. 
[2] F.S. Apple, M.M. Murakami et al., Predictive value of cardiac troponin I and $\mathrm{T}$ for subsequent death in end-stage renal disease, Circulation 106 (2002), 2941-2945.

[3] F.S. Apple, A.H. Wu et al., European Society of Cardiology and American College of Cardiology guidelines for redefinition of myocardial infarction: how to use existing assays clinically and for clinical trials, Am Heart J 144 (2002), 981-986.

[4] P. Ardelt, P. Dorka et al., Microanalysis and distribution of cardiac troponin I phospho species in heart areas, Biol Chem 379 (1998), 341-347.

[5] A.H. Association, Heart Disease and Stroke Statistics - 2004 Update, A. H. Association.

[6] U. Bilitewski, M. Genrich et al., Biochemical analysis with microfluidic systems, Anal Bioanal Chem 377 (2003), 556569.

[7] H. Brzeski, R.A. Katenhusen et al., Albumin depletion method for improved plasma glycoprotein analysis by twodimensional difference gel electrophoresis, Biotechniques 35 (2003), 1128-1132.

[8] D.J. Cahill, E. Nordhoff, Protein arrays and their role in proteomics, Adv Biochem Eng Biotechnol 83 (2003), 177-187.

[9] D.A. Colantonio, W. Pickett et al., Detection of cardiac troponin I early after onset of chest pain in six patients, Clin Chem 48 (2002), 668-671.

[10] T.J. Cornish, R.J. Cotter, A curved-field reflectron for improved energy focusing of product ions in time-of-flight mass spectrometry, Rapid Commun Mass Spectrom 7 (1993), 10371040 .

[11] B. Dayal, N.H. Ertel, ProteinChip technology: a new and facile method for the identification and measurement of highdensity lipoproteins apoA-I and apoA-II and their glycosylated products in patients with diabetes and cardiovascular disease, J Proteome Res 1 (2002), 375-380.

[12] Ding, R. Labugger et al., Development of an Interaction Discovery Mapping? Methodology for Assessing Myocardial Infarction Severity using SELDI-TOF Mass Spectrometry, A.A.C.C.

[13] E. Gianazza, P. Arnaud, Chromatography of plasma proteins on immobilized Cibacron Blue F3-GA. Mechanism of the molecular interaction, Biochem J 203 (1982), 637-641.

[14] I. Giuliani, J.P. Bertinchant et al., Determination of cardiac troponin I forms in the blood of patients with acute myocardial infarction and patients receiving crystalloid or cold blood cardioplegia, Clin Chem 45 (1999), 213-222.

[15] I. Giuliani, J.P. Bertinchant et al., Determination of cardiac troponin I forms in the blood of patients with unstable angina pectoris, Clin Biochem 35 (2002), 111-117.

[16] N.I. Govorukhina, A. Keizer-Gunnink et al., Sample preparation of human serum for the analysis of tumor markers. Comparison of different approaches for albumin and gammaglobulin depletion, J Chromatogr A 1009 (2003), 171-178.

[17] C.B. Granger, J.E. Van Eyk et al., NHLCI clinical proteomics working group report, Circulation In Press, 2004.

[18] W.J. Henzel, T.M. Billeci et al., Identifying proteins from two-dimensional gels by molecular mass searching of peptide fragments in protein sequence databases, Proc Natl Acad Sci USA 90 (1993), 5011-5015.

[19] A.G. Katrukha, A.V. Bereznikova et al., Degradation of cardiac troponin I: implication for reliable immunodetection, Clin Chem 44 (1998), 2433-2440.

[20] L.J. Kricka, T. Joos et al., Protein microarrays: a literature survey, Clin Chem 49 (2003), 2109.
[21] R. Labugger, L. Organ et al., Extensive troponin I and T modification detected in serum from patients with acute myocardial infarction, Circulation 102 (2000), 1221-1226.

[22] R. Labugger, J.A. Simpson et al., Strategy for analysis of cardiac troponins in biological samples with a combination of affinity chromatography and mass spectrometry, Clin Chem 49 (2003), 873-879.

[23] P. Lecchi, A.R. Gupte et al., Size-exclusion chromatog raphy in multidimensional separation schemes for proteome analysis, J Biochem Biophys Methods 56 (2003), 141-152.

[24] Y. Lee, E.K. Lee et al., ProteoChip: a highly sensitive protein microarray prepared by a novel method of protein immobilization for application of protein-protein interaction studies, Proteomics 3 (2003), 2289-2304.

[25] A. Lueking, A. Possling et al., A Nonredundant Human Protein Chip for Antibody Screening and Serum Profiling, Mol Cell Proteomics 2 (2003), 1342-1349.

[26] K. Millea, I. Krull, Subproteomics in Analytical Chemistry: Chromatographic Fractionation Techniques in the Characterization of Proteins and Peptides, J Liq Chromatogr 26 (2003), 2195-2224.

[27] G.J. Opiteck, S.M. Ramirez et al., Comprehensive twodimensional high-performance liquid chromatography for the isolation of overexpressed proteins and proteome mapping, Anal Biochem 258 (1998), 349-361.

[28] Z. Ouyang, Z. Takats et al., Preparing protein microarrays by soft-landing of mass-selected ions, Science 301 (2003), 13511354.

[29] R. Pieper, Q. Su et al., Multi-component immunoaffinity subtraction chromatography: an innovative step towards a comprehensive survey of the human plasma proteome, Proteomics 3 (2003), 422-432.

[30] A. Ressine, S. Ekstrom et al., Macro-/nanoporous silicon as a support for high-performance protein microarrays, Anal Chem 75 (2003), 6968-6974.

[31] J.P. Salier, P. Rouet et al., The inter-alpha-inhibitor family: from structure to regulation, Biochem J 315(Pt 1) (1996), 1-9.

[32] S.Y. Seong, C.Y. Choi, Current status of protein chip development in terms of fabrication and application, Proteomics 3 (2003), 2176-2189.

[33] Y. Shen, R.D. Smith, Proteomics based on high-efficiency capillary separations, Electrophoresis 23 (2002), 3106-3124.

[34] J.A. Simpson, S. Iscoe et al., Myofilament proteomics: unraveling contractile dysfunction, in: Genomic and Proteomic Analysis of Cardiovascular Disease: molecular mechanism, therapeutic targets and diagnostics, Wiley and Son, eds, Germany, 2003, pp. 317-342.

[35] L.F. Steel, M.G. Trotter et al., Efficient and specific removal of albumin from human serum samples, Mol Cell Proteomics 2 (2003), 262-270.

[36] N. Tang, P. Tornatore et al., Current developments in SELDI affinity technology, Mass Spectrom Rev 23 (2004), 34-44.

[37] Z.H. Wang, G. Jin, A label-free multisensing immunosensor based on imaging ellipsometry, Anal Chem 75 (2003), 61196123.

[38] A.H. Wu, Y.J. Feng et al., Characterization of cardiac troponin subunit release into serum after acute myocardial infarction and comparison of assays for troponin T and I. American Association for Clinical Chemistry Subcommittee on cTnI Standardization, Clin Chem 44 (1998), 1198-1208.

[39] R. Zhang, L. Barker et al., Mining biomarkers in human sera using proteomic tools, Proteomics 4 (2004), 244-256. 


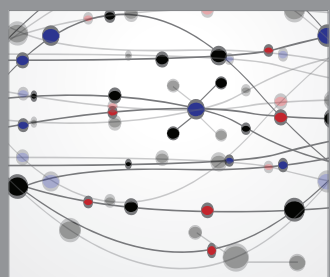

The Scientific World Journal
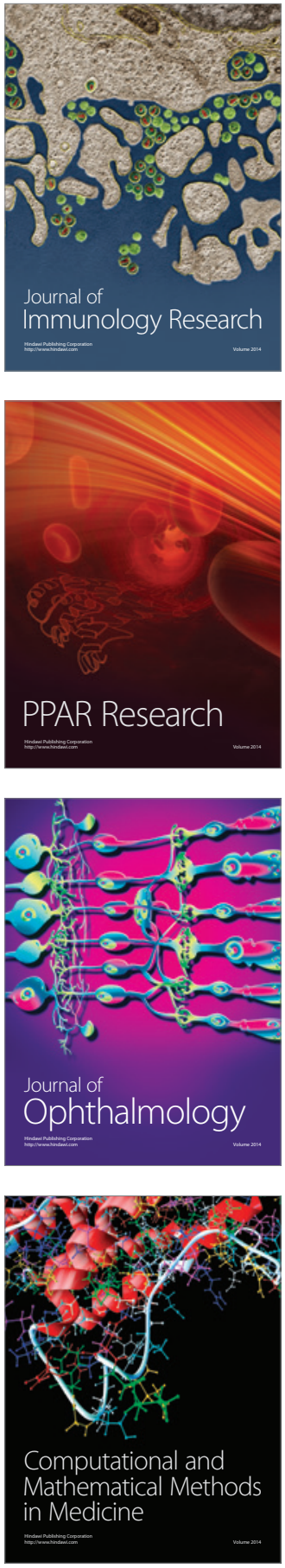

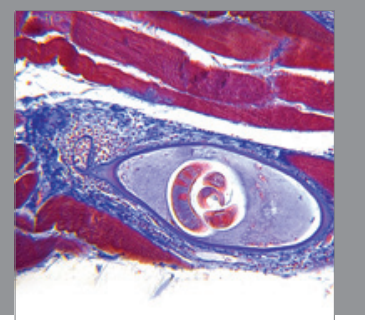

Gastroenterology

Research and Practice
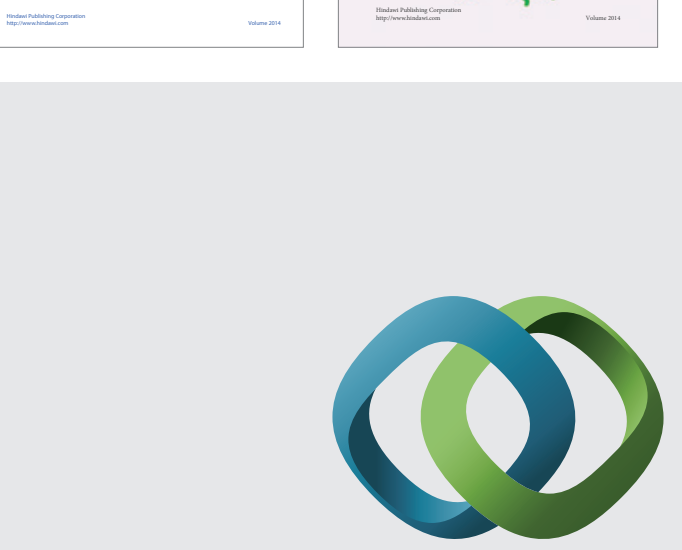

\section{Hindawi}

Submit your manuscripts at

http://www.hindawi.com
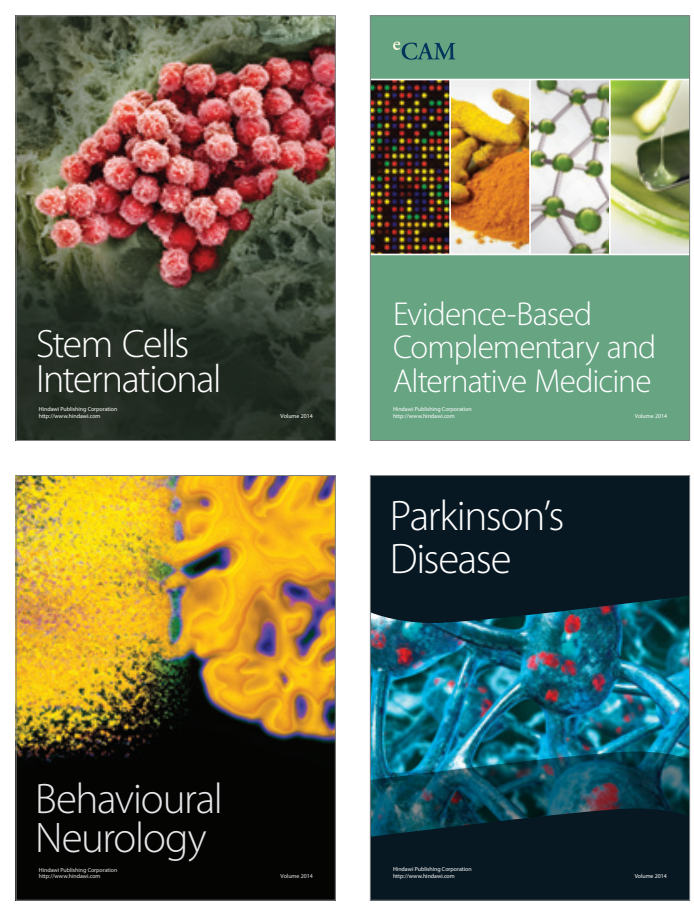

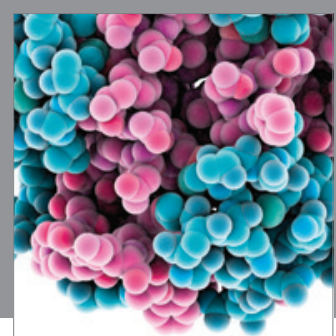

Journal of
Diabetes Research

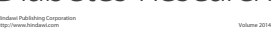

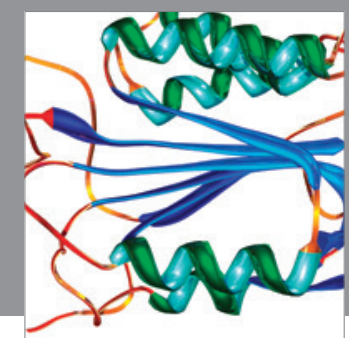

Disease Markers
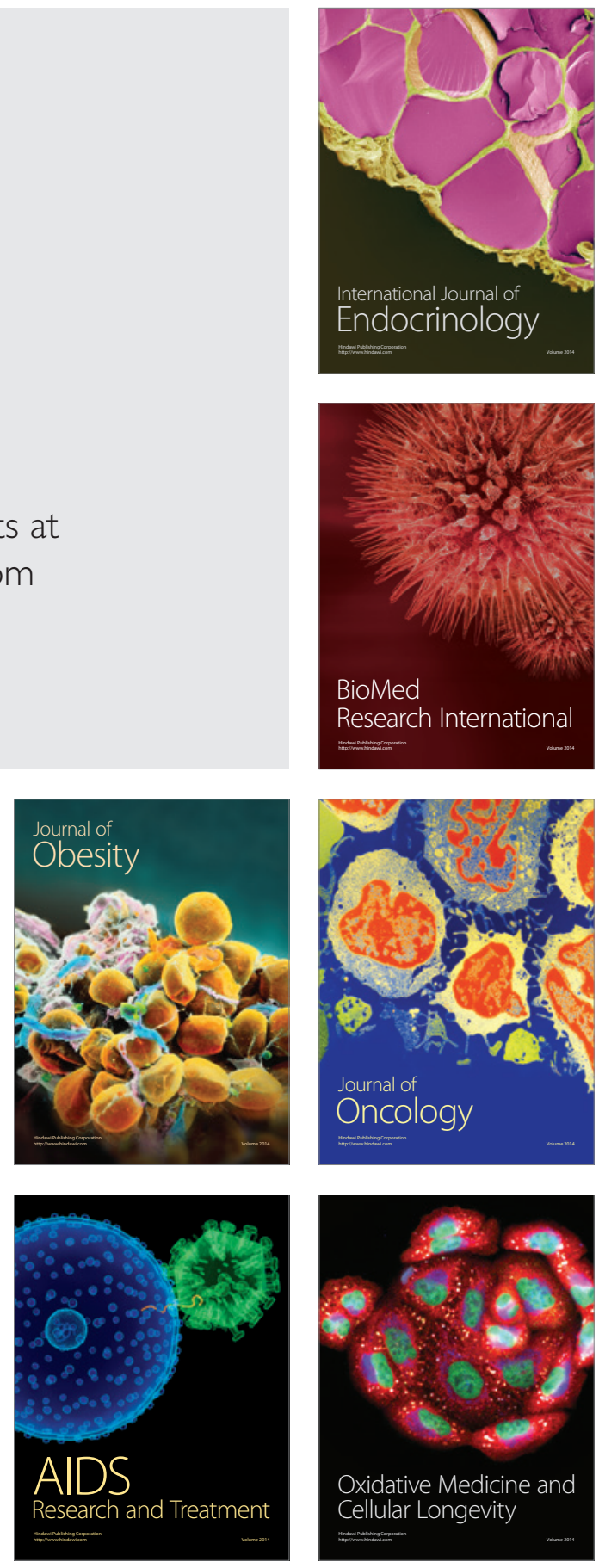